\title{
Phytoprotection
}

\section{Note du rédacteur en chef}

\section{Daniel Dostaler}

Volume 90, numéro 2, août 2009

URI : https://id.erudit.org/iderudit/044020ar

DOI : https://doi.org/10.7202/044020ar

Aller au sommaire du numéro

Éditeur(s)

Société de protection des plantes du Québec (SPPQ)

ISSN

0031-9511 (imprimé)

1710-1603 (numérique)

Découvrir la revue

Citer ce document

Dostaler, D. (2009). Note du rédacteur en chef. Phytoprotection, 90(2), 45-45.

https://doi.org/10.7202/044020ar d'utilisation que vous pouvez consulter en ligne.

https://apropos.erudit.org/fr/usagers/politique-dutilisation/ 


\section{Note du rédacteur en chef}

La parution du Volume 89 (2-3) de Phytoprotection porte des fruits. Monsieur Marcel Mailloux, agronomeentomologiste retraité du ministère de l'Agriculture, des Pêcheries et de I'Alimentation du Québec (MAPAQ), nous a fait parvenir un précieux complément de l'histoire de l'entomologie agricole parue dans Phytoprotection 89 (2-3) à I'occasion du centenaire de la Société de protection des plantes du Québec. Marcel Mailloux porte à notre connaissance que parmi les laboratoires de terrain créés par le MAPAQ, qui s'appelait alors le ministère de l'Agriculture, celui de Farnham, ouvert en 1949, était connu sous l'appellation "Station de protection des vergers". Cette station visait par ses travaux, bien sûr, la biologie et la lutte aux tétranyques du pommier (Cloutier et al. 2008. Phytoprotection 89: 79-97), mais aussi, de façon plus globale à partir des années 1975-80, le dépistage des ravageurs de la pomme et la conception de programmes de lutte intégrée dans les vergers de pommiers, une première au Québec parmi toutes les productions végétales. Les recherches ${ }^{1}$ auxquelles a contribué Marcel Mailloux ont mené en particulier à l'élaboration de modèles informatisés de prévision des stades de développement des ravageurs et de la phénologie du pommier, le tout en relation avec la climatologie. Ces modèles novateurs, améliorés au fil du temps, servent encore dans le contexte des avertissements phytosanitaires du Réseau pommier, dont Marcel Mailloux a été le premier avertisseur (Bernier et al. 2008. Phytoprotection 89: 143-164). Un grand merci à Marcel Mailloux pour ce témoignage et ces précisions.

Daniel Dostaler

Rédacteur en chef

Marcel Mailloux (M.Sc. 1960, Université McGill). Agronome et entomologiste. Membre honoraire de la Société de protection des plantes du Québec et Membre émérite de la Société d'entomologie du Québec.

\footnotetext{
${ }^{1}$ Exemples de publications:
}

- Rivard, I. et M. Mailloux. 1974. Grapholitha prunivora (Walsh) dans les pommeraies du sud-ouest du Québec. Phytoprotection 55: 29-32.

- Mailloux, M. 1984. Le réseau d'avertissements phytosanitaires du pommier au Québec. Pages 177-188 dans C. Vincent et N.J. Bostanian (éds.), La phytoprotection des vergers de pommiers au Québec. Bull. tech. no. 19, Agriculture Canada, Station de recherche, Saint-Jean-sur-Richelieu, Québec.

- Vincent, C. et M. Mailloux. 1988. Abondance, importance des dommages et distribution de l'hoplocampe des pommes au Québec de 1979 à 1986. Ann. Soc. Entomol. Fr. 24: 39-46.

- Vincent, C., M. Mailloux et A.E.C. Hagley. 1986. Nonsticky pheromone-baited traps for monitoring the spotted tentiform leafminer (Lepidoptera: Gracillariidae). J. Econ. Entomol. 79: 1666-1670.

- Vincent, C., M. Mailloux, E.A.C. Hagley, W.H. Reissig, W.M. Coli et T.A. Hosmer. 1990. Monitoring the codling moth (Lepidoptera: Olethreutidae) and the obliquebanded leafroller (Lepidoptera: Tortricidae) with sticky and nonsticky traps. J. Econ. Entomol. 83: $434-440$. 